\title{
Acute and chronic exposure to ethanol and the electrophysiology of the brush border membrane of rat small intestine
}

\author{
F AL-BALOOL, E S DEBNAM, AND R MAZZANTI \\ From the Department of Physiology, Royal Free Hospital School of Medicine, London
}

SUMMARY In this study we have investigated the effects of (a) chronic ethanol intake on glucose and galactose absorption across the rat jejunum in vivo and on the potential difference across the isolated brush border membrane $\left(V_{m}\right)$ and $(b)$ acute exposure to ethanol $(4 \%$ or $8 \%)$ and acetaldehyde $(0 \cdot 25 \%)$ on changes in $\mathrm{V}_{m}$ associated with $\mathrm{Na}^{+}$-dependent galactose absorption across the jejunum and ileum. Chronic ethanol intake was associated with hyperpolarisation of $V_{m}$ and an enhanced galactose but not glucose transport. Acute ethanol and acetaldehyde were without effect on $V_{m}$ whether or not galactose was present. We conclude that while a greater electrochemical gradient across the brush border membrane is a likely explanation for the stimulation of galactose absorption induced by ethanol feeding, factors other than changes in $V_{m}$ are responsible for the inhibitory effects of acute ethanol.

It is well known that acute exposure of the intestinal mucosa to ethanol reduces the transport of those nutrients which use the $\mathrm{Na}^{+}$-gradient for movement across the brush border membrane (BBM) ${ }^{1-3}$ one of which is the hexose galactose. ${ }^{+5}$ In marked contrast, we have noted an enhanced galactose absorption in rats maintained for four weeks on a liquid diet containing 5\% ethanol, ${ }^{\circ}$ a finding which is a likely consequence of an early maturation of enterocytes.?

The potential difference across the brush border membrane $\left(V_{m}\right)$ is an important driving force for $\mathrm{Na}^{+}$-dependent sugar movement into enterocytes. ${ }^{\circ}$ In order to examine further the mechanisms involved in the functional response to acute and chronic exposure to ethanol, we have compared the effects of ethanol feeding on the active absorption of glucose and galactose in vivo and report those changes in $\mathrm{V}_{\mathrm{m}}$, measured in vitro, associated with active galactose uptake after acute or chronic exposure to ethanol. Finally, because acetaldehyde, an intestinal metabolite of ethanol, inhibits $\mathrm{Na}^{+}$-dependent sugar movement across the brush border membrane, ${ }^{2}$ we have assessed the effect of acetaldehyde on $V_{m}$.

Address for correspondence: Dr E S Debnam. Department of Physiology. Royal Free Hospital School of Medicine, Rowland Hill Street, London NW3 2PF.

Accepted for publication 15 March 1989

\section{Methods}

DIETS AND ANAESTHESIA

Sprague-Dawley rats (RFHSM bred, initial weight $140-150 \mathrm{~g}$ ) were individually caged and pair-fed for 26-32 days on a liquid control diet containing Complan (Farley Health Products, Nottinghamshire, UK), casein, glucose, and corn oil to provide $34 \%$ of the total calories as carbohydrate, $18 \%$ as proteins and the remainder as fat." For the alcohol diet, ethanol $(5 \% \mathrm{w} / \mathrm{v})$ was substituted for a proportion of the fat content so as to provide $36 \%$ of the total calories. Additional vitamins (Orovite 7, Beecham, Brentford, UK) $5 \mathrm{~g} / \mathrm{l}$ were added to each diet to satisfy the requirements for growth."' The alcohol diet was replaced by the control diet 18 hours before experimentation. Chow fed animals $(200-250 \mathrm{~g})$ were maintained on Diet 41B (Grain Harvesters Ltd, Kent, UK) up to the time of use.

Pentobarbitone sodium ( $90 \mathrm{mg} / \mathrm{kg}$ ip, Sagatal, May and Baker, Essex, UK), was used for in vivo absorption studies and to enable tissue to be removed for in vitro experiments.

MEASUREMENTS OF $\mathrm{V}_{\mathrm{m}}$

Full details of the in vitro preparation used, the techniques for the preparation and use of microelectrodes and the criteria adopted for accepting 
impalements have been previously described." Jejunal tissue was taken approximately $10 \mathrm{~cm}$ from the ligament of Treitz and ileal tissue approximately $10 \mathrm{~cm}$ from the ilealcaecal junction.

\section{SUGAR ABSORPTION IN VIVO}

The techniques used to measure glucose and galactose absorption in vivo are similar to those described previously. ${ }^{12}$ In brief, uptake was determined by circulating the sugar $(64 \mathrm{mM})$ dissolved $\left.\begin{array}{llll}\text { in gassed }(95 \% & \mathrm{O}_{2}: 5 \% & \mathrm{CO}_{2}\end{array}\right)$ bicarbonate saline through a $25 \mathrm{~cm}$ length of upper jejunum. After 30 minutes, the perfusate in the intestine and circulation system was washed out, deproteinised and estimated for either glucose or galactose..$^{13}$ The segment of intestine was removed and its length measured. Sugar uptake was calculated as luminal loss and expressed as $\mathrm{nmol} / \mathrm{cm} / \mathrm{min}$. Uptake data were corrected for non-saturable absorption ${ }^{12}$ in order to obtain data for active, $\mathrm{Na}^{+}$-dependent transport.

\section{STATISTICAL ANALYSIS}

Data are given as mean (SE). Difference between means were evaluated by Student's $t$ test for unpaired samples. Differences between the magnitude of the depolarisation obtained upon addition of galactose to the mucosal fluid were compared using an Interaction test. ${ }^{14}$ For both tests, differences were considered not significant at $\mathrm{p}>0 \cdot 05$.

\section{CHEMICAIS}

D-galactose (glucose free) and phlorhizin were obtained from Sigma UK. Absolute ethanol was purchased from James Burrough, Essex, UK. All other chemicals were Analar Grade from BDH, Dorset, UK.

\section{Results}

The data for ethanol intake and body weight for animals on the two liquid diets over the 26-31 day feeding period are shown in Figure 1. The gain in body weight per day was very similar in control $(4.5$ $(0 \cdot 23) \mathrm{g} /$ day $)(\mathrm{n}=8)$ and ethanol fed animals $(4.76$ $(0 \cdot 27) \mathrm{g} /$ day $)(n=9)$ over the second half of the feeding period during which time ethanol consumption expressed per $\mathrm{kg}$ body weight was remarkably constant. The initial weight loss in the ethanol group may be because of the diuretic effect of ethanol. No evidence of diarrhoea was apparent in rats fed either diet.

$V_{m}$ AFTER CHRONIC ETHANOLINGESTION Approximately $15 \%$ of impalements resulted in electrical stability for a minimum period of 10 seconds. Examples, showing the profiles of three

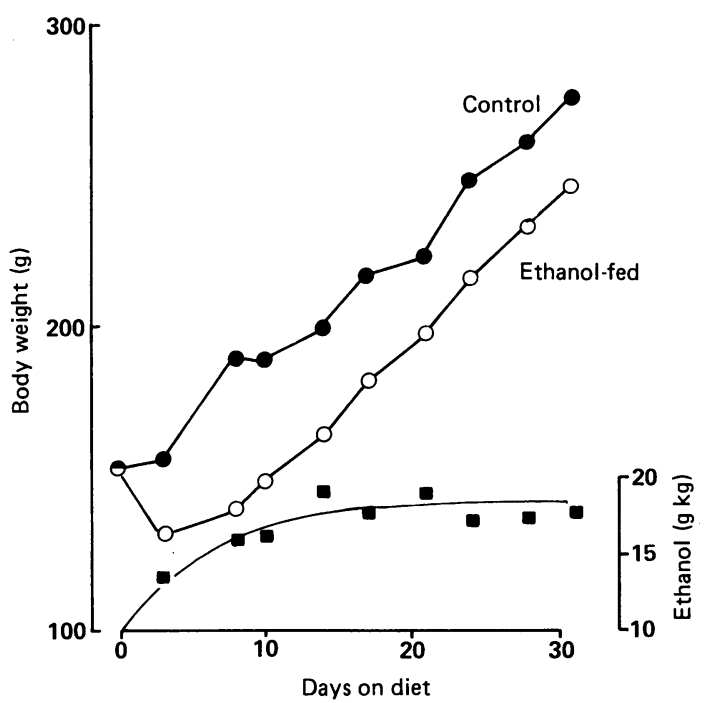

Fig. 1 Body weight in ethanol fed and pair fed control rats. Also shown is the amount of ethanol consumed expressed as $\mathrm{g} / \mathrm{kg}$ body weight at various times over the 32 day feeding period.

successful impalements whilst exposing jejunum from chow-fed rats to oxygenated bicarbonate buffer are shown in Figure 2. In all cases, an immediate deflection occurred upon microelectrode penetration of the cell and the potential difference returned to its original baseline following withdrawal of the electrode giving a mean potential difference of $-43 \cdot 7(2 \cdot 1) \mathrm{mV}(\mathrm{n}=36)$. This value is similar to previously reported measurements of $\mathrm{V}_{\mathrm{m}}$ in rat small intestine. ${ }^{1 / n}$ The time between removal of tissue from the animal and exposure to oxygenated buffer never exceeded one minute as further delays affect

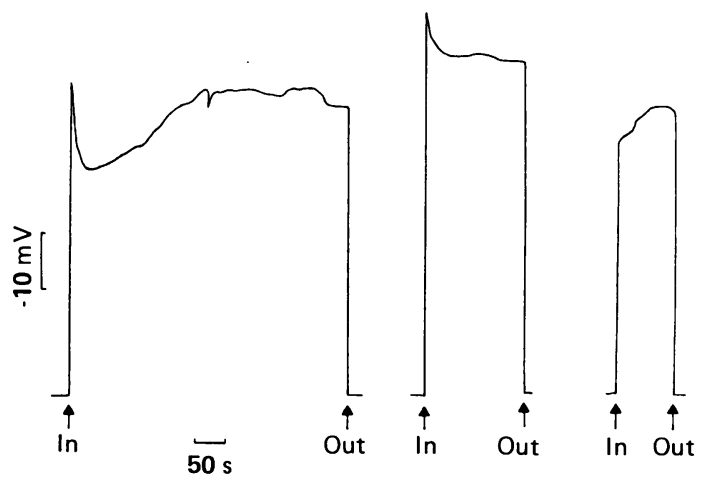

Fig. 2 Three examples of changes in potential difference observed during microelectrode impalement of enterocytes. Arrows refer to impalement and withdrawal of the microelectrode. In each case, electrical stability was maintained for a minimum of 10 seconds. 
Table 1 Potential difference across the brush border membrane $\left(V_{\mathrm{m}}\right)$ in control and ethanol fed rats

\begin{tabular}{llll}
\hline & \multicolumn{3}{c}{$V_{\mathrm{m}}(\mathrm{mV})$} \\
\cline { 2 - 4 } & - Galactose & + (ialactose $(10 \mathrm{mM})$ & $p$ \\
\hline Control diet & $-42.9(1 \cdot 5)[81]$ & $-34 \cdot 4(1 \cdot 2)[61]$ & $<0.001$ \\
Ethanol diet & $-51 \cdot 2(1.5)[79]$ & $-36 \cdot 1(1.4)[6(0)$ & $<0.001$ \\
$\mathrm{p}$ & $<0 .(0) 1$ & $>0.05$ & \\
\hline
\end{tabular}

Values are given as mean (SEM) with number of impalements in square brackets. At least three animals were used for each group of experiments.

tissue viability. ${ }^{15}$ For non-gassed tissue, $V_{m}$ was found to be $-7 \cdot 6(1 \cdot 3) \mathrm{mV}(\mathrm{n}=7)$.

Ethanol feeding resulted in a significant hyperpolarisation of the brush border membrane of some $8.3 \mathrm{mV}$ (Table 1); the distribution of individual impalements in intestine from control and ethanol fed rats in shown in Figure 3. The addition of Dgalactose $(10 \mathrm{mM})$ to the mucosal fluid caused a depolarisation of $8.5 \mathrm{mV}$ and $15.1 \mathrm{mV}$, respectively, in intestine from control and ethanol fed rats $(\mathrm{p}<0 \cdot 05)$ (Table 1$)$.

The effects of $\mathrm{Na}^{+}$concentration on $\mathrm{V}_{\mathrm{m}}$ was studied by using mucosal solutions of varying $\mathrm{Na}^{\prime}$ concentration, mannitol being used to maintain isotonicity. A comparison of the response using intestine from control and ethanol fed animals is shown in Figure 4. Under control conditions, $\mathrm{Na}^{+}$ concentration had a profound effect on the magnitude of $V_{m}$ and analysis of the region between

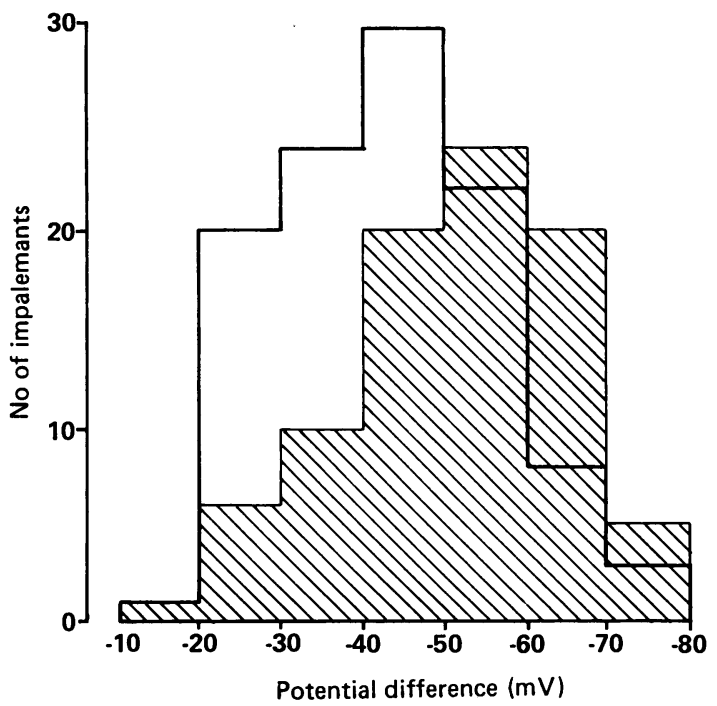

Fig. 3 Histogram showing the distribution of measured values of $V_{\mathrm{m}}$ in intestine from control (clear area) and ethanol fed (shaded area) rats.

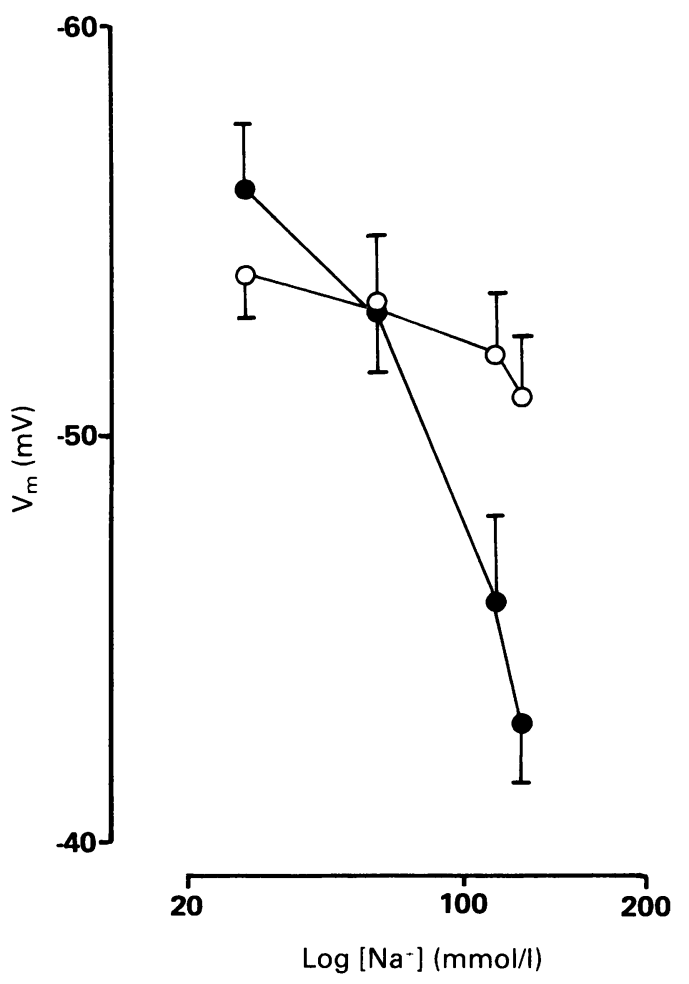

Fig. 4 Effect of chronic ethanol ingestion on the relationship between $V_{\mathrm{m}}$ and mucosal sodium concentration. Animals were maintained on either ethanol $(O)$ or control liquid diet (O). Means (SEM) are given with 15-81 impalements for each mean value.

61 and $143 \mathrm{mM} \mathrm{Na}^{+}$revealed that the slope of the response for a 10 -fold change in $\mathrm{Na}^{\prime}$ was $25.5 \mathrm{mV}$. After ethanol, however, a decrease in $\mathrm{Na}$ ' from 143 to $25 \mathrm{mM}$ was without significant effect on $\mathrm{V}_{\mathrm{m}}$ $(\mathrm{p}>0 \cdot 05)$.

\section{ACUTE EXPOSURE: TO ETHANOI. AND}

ACETALDEHYDE

Jejunal and ileal tissue from chow fed rats was used to study the effect of acute exposure to ethanol $(4 \%$ or $8 \% \mathrm{w} / \mathrm{v})$ and acetaldehyde $(0 \cdot 25 \% \mathrm{w} / \mathrm{v})$ on $\mathrm{V}_{\mathrm{m}}$. At these concentrations, these agents have been shown to inhibit sugar transport across the BBM.: In their absence, $V_{m}$ was very similar to that obtained from tissue exposed to the control liquid diet $(p>0.5$, Tables 1,2$)$. In contrast with the hyperpolarisation seen after chronic ethanol feeding, however, the presence of ethanol or acetaldehyde in the mucosal fluid were without effect on $\mathrm{V}_{\mathrm{m}}$ and, furthermore, these agents did not influence the depolarisation of $\mathrm{V}_{\mathrm{m}}$ induced by the presence of D-galactose in the mucosal fluid (jejunum: $4 \%$ ethanol $p>0 \cdot 5,8 \%$ 
Table 2 Acute effect of ethanol and acetaldehyde on $V_{\mathrm{m}}$ of jejunum and ileum

\begin{tabular}{|c|c|c|c|c|}
\hline & \multicolumn{2}{|c|}{ Jejunum } & \multicolumn{2}{|c|}{ lle'um } \\
\hline & No galactose & + Galactose $(10 \mathrm{mM})$ & No galactose' & + Galactose $(10 \mathrm{mM})$ \\
\hline $\begin{array}{l}\text { Bicarbonate buffer } \\
+ \text { Ethanol }(4 \%) \\
+ \text { Ethanol }(8 \%) \\
+ \text { Acetaldehyde }(0.25 \%)\end{array}$ & $\begin{array}{l}-43.7(2 \cdot 1)[36] \\
-43 \cdot 8(1.9)[62]^{*} \\
-43 \cdot 1(1 \cdot 2)[80]^{*} \\
-41 \cdot 6(1 \cdot 1)[82]^{*}\end{array}$ & $\begin{array}{l}-38.4(1 \cdot 5)[42]^{\dagger} \\
-36 \cdot 4(1 \cdot 3)[49]^{* \dagger} \\
-33 \cdot 6(1 \cdot 4)[41]^{* \dagger} \\
-35.2(1 \cdot 3)[42]^{* \dagger}\end{array}$ & $\begin{array}{l}-4(1.5(1.1)[6.5] \\
-42.7(1.5)[5()]^{*} \\
-39 \cdot 2(1.1)[44]^{*} \\
-41.8(1.3)[38]^{*}\end{array}$ & $\begin{array}{l}-36 \cdot 6(1 \cdot 1)[4.3]^{\dagger} \\
-36 \cdot 4(1 \cdot 1)[49]^{*} \dagger \\
-36 \cdot 8(1 \cdot 5)[38]^{*} \\
-3.5 \cdot 8(1 \cdot 6)\left[\left.32\right|^{* \dagger}\right.\end{array}$ \\
\hline
\end{tabular}

Tissue was obtained from chow-fed rats and values $(\mathrm{mV})$ are given as mean (SEM) with number of impalements in square brackets. At least three animals were used for each group of experiments. ${ }^{*} p>0.05$ compared with $V_{m}$ in the presence of bicarbonate buffer alone: $\nmid p<0.05$ compared with $V_{m}$ in the absence of galactose.

ethanol $p>0 \cdot 2$, acetaldehyde $p>0 \cdot 7$; ileum: $4 \%$ ethanol $p>0 \cdot 05,8 \%$ ethanol $p>0 \cdot 9$, acetaldehyde $\mathrm{p}>0 \cdot 1)$.

SUGAR ABSORPTION IN VIVO

Chronic ethanol feeding resulted in a $31.3 \%$ stimulation of active galactose absorption ( $p<0 \cdot 05$, Fig. 5), but was without significant effect on glucose absorption $(\mathrm{p}>0 \cdot 4)$.

Values for glucose and galactose absorption in rats maintained on the liquid control diet were very similar to jejunal uptakes using chow fed animals (glucose: $398.1(31.0) \mathrm{nmol} / \mathrm{cm} / \mathrm{min}(\mathrm{n}=6)$; galactose: $317 \cdot 4(26 \cdot 5) \mathrm{nmol} / \mathrm{cm} / \mathrm{min}(\mathrm{n}=6)$.

\section{Discussion}

Previous studies in the rat on the functional effects of acute ethanol exposure on intact intestinal mucosa or isolated brush border membrane (BBM) have reported a diminished active sugar uptake. ${ }^{1-3}$ In contrast, there is conflicting information on the response to chronic ethanol ingestion on hexose absorption. ${ }^{17}$ The discrepancies between the various studies are likely to be caused by such variables as the time scale of ethanol ingestion and the alcohol and carbohydrate composition of the control and ethanol diets. A crucial factor of the dietary regime adopted in this study was that the control diet used fat as a calorie substitute for ethanol. Carbohydrate substitution was avoided as variations in the dietary level of this nutrient have been shown to influence sugar uptake. ${ }^{1 \mathrm{x}}$ Animals on control and experimental diets gained weight at a similar rate and this, together with our previous observations of an unchanged villus height and enterocyte number after ethanol," suggests that both groups were of a comparable nutritional state. It must also be emphasised that rats were not subject to overnight starvation before experimentation as this procedure is associated with profound changes in $\mathrm{V}_{\mathrm{m}}$ and sugar absorption."119 The ethanol concentrations used for both chronic and acute studies were within the range found in the upper intestine of man after moderate alcohol ingestion."

For in vivo absorption studies, a sugar concentration of $64 \mathrm{mM}$ was chosen as in control animals, uptake at this concentration gives a good approxima-

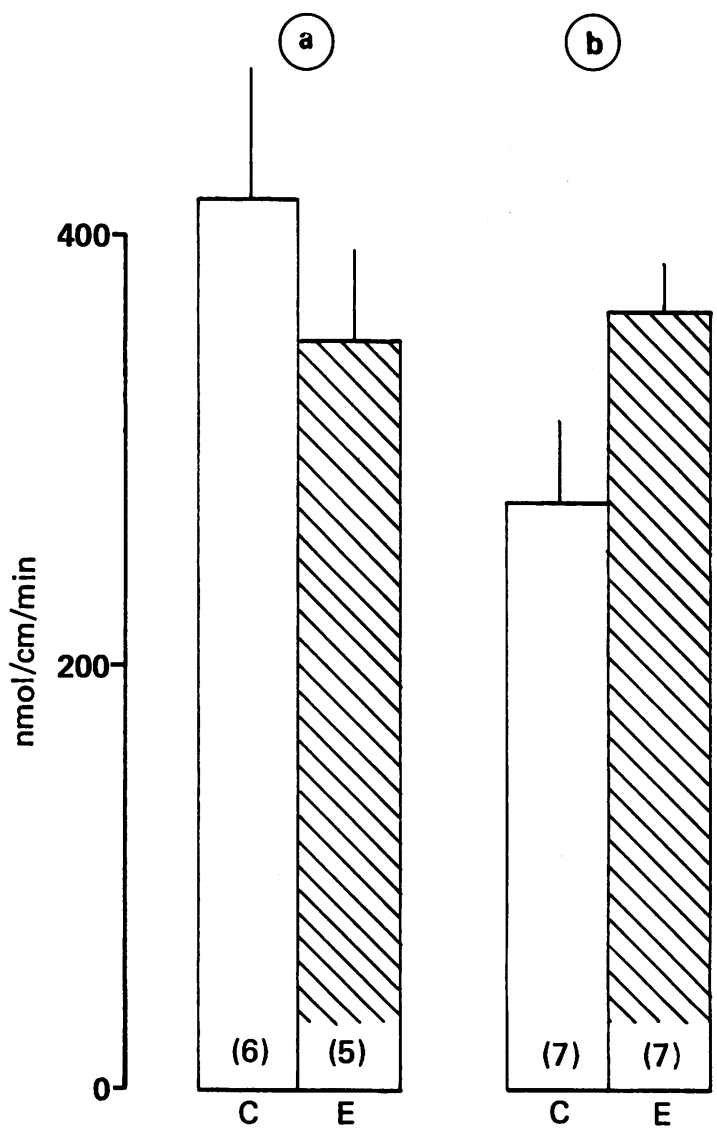

Fig. 5 Glucose (a) and galactose (b) absorption in vivo in rats fed control (C) or ethanol (E) liquid diets. Data have been corrected for the linear uptake process (see Methods) and are given as means (SEM) with number of observations in parentheses. 
tion of $\mathrm{V}_{\max } \cdot{ }^{12}$ Hypertonicity of the circulating sugar containing buffer is unavoidable because even modest reductions in $\mathrm{Na}^{+}$concentration to compensate for this osmolality change cause a significant inhibition of absorption. ${ }^{21}$ Our results confirm and extend a previous report of an enhanced capacity for active galactose absorption in rats maintained on ethanol. ${ }^{\circ}$

The differential effect of ethanol feeding on galactose and glucose absorption may reflect separate carrier systems for the two sugars."2 This observation may also be a consequence of the ability of enterocytes to metabolise glucose ${ }^{23}$ and it is of interest that a previous study has reported a greater inhibition, by ethanol, of glucose compared with galactose absorption. ${ }^{+}$The use of galactose, a nonmetabolisable sugar ${ }^{2.3}$ to assess changes in intestinal uptake avoids problems of data interpretation imposed by intracellular metabolism of absorbed sugar and alterations in galactose uptake after ethanol give a better indication of changes in membrane transport perse.

The stability and magnitude of $\mathrm{V}_{\mathrm{m}}$ measurements in our study were a consequence of rapid mounting and oxygenation of the isolated tissue. It has been argued elsewhere ${ }^{15}$ that tissue anoxia is a likely cause of the low $\mathrm{V}_{\mathrm{m}}$ values obtained previously. As the magnitude of the electrical gradient across the BBM is an important driving force for active sugar entry into the enterocyte, ${ }^{x}$ our finding of an enhanced $V_{m}$ following ethanol feeding provides an obvious explanation for the stimulation of galactose absorption in vivo.

The process of enterocyte migration along the jejunal villi is associated with hyperpolarisation of $\mathrm{V}_{\mathrm{m}},{ }^{2-4}$ and we have previously suggested that the adaptation to chronic ethanol ingestion may be related to a reduced enterocyte transit resulting in a higher proportion of mature cells on the villus." In this context, it is of interest that prolonged ethanol feeding is associated with an increased lactase activity $y^{\mathrm{h}}$ and a stimulation of iron absorption ${ }^{25}$ but no change in villus height or enterocyte number."

Data obtained in $\mathrm{Na}$ ' replacement experiments in this present study imply that a reduction in $\mathrm{Na}^{+}$permeability of the brush border membrane is responsible, at least in part, for the hyperpolarisation seen in ethanol fed rats. The electrical response is unlikely to be the result of alterations in $\mathrm{Cl}^{-}$concentration of the mucosal fluid during these experiments, as $\mathrm{Cl}$ diffusion makes no contribution to $\mathrm{V}_{\mathrm{m}} \cdot{ }^{15}$ Interestingly, membrane hyperpolarisation together with an increased $\mathrm{Na}^{\circ}$-dependent nutrient uptake occur in two other experimental conditions, fasting and hyperglucagonacmia, ${ }^{20} 27$ a reduced enterocyte transit being a recognised feature of both conditions.
Acute exposure to ethanol at concentrations similar to, or below, those used in this present work have been shown to inhibit $\mathrm{Na}$-dependent galactose transport. ${ }^{+5}$ As we were unable to detect changes in $\mathrm{V}_{\mathrm{m}}$ after acute exposure to ethanol, previous explanations of the inhibition of active nutrient uptake by ethanol or its intestinal metabolite acetaldehyde $^{2}$ based on an increased sodium conductance of the $\mathrm{BBM}^{1}$ : can be eliminated as depolarisation of $\mathrm{V}_{\mathrm{m}}$ would be expected in the presence of these agents. The precise mechanism of the inhibitory effect of acute ethanol on brush border membrane sugar transport must await further studies, but the osmotic effects of ethanol can be excluded.' Other possibilities include direct conformational effects on the hexose transporter and increased fluidity of the BBM. ${ }^{2 \times}$

In conclusion, we have shown that the jejunal epithelium of ethanol fed rats displays an enhanced ability for galactose transport, the likely explanation being a higher electrical driving force for $\mathrm{Na}^{+}-$ coupled movement across the BBM. The significance of our findings to absorption studies in alcoholics is unclear because an associated poor nutritional state will complicate the interpretation of uptake data. There is, however, some evidence for an enhanced absorption of glucose and xylose in well nourished alcoholics." The chronic effects of ethanol resulting from a slower enterocyte transit may compensate, at least in part, for its acute inhibitory action on sugar uptake.

We are grateful to the University of Kuwait and the Smith Kline Foundation for financial support of this work.

\section{References}

1 Beesley RC. Ethanol inhibits $\mathrm{Na}^{\text {-gradient dependent }}$ uptake of L-amino acids into intestinal brush border membrane vesicles. Dig Dis Sci 1986: 31: 987-92.

2 Tillotson LG, Carter EA. Inue K-I, Isselbacher KJ. Inhibition of $\mathrm{Na}^{-}$-stimulated glucose transport function and perturbation of intestinal microvillus membrane vesicles by ethanol and acetaldehyde. Arch Biochem Biophys 1981; 207: 360-70.

3 Chang T. Lewis J, Glazko AJ. Effect of ethanol and other alcohols on the transport of amino acids and glucose by everted sacs of rat small intestine. Biochim Biophys Acta 1967: 135: 10(0)-7.

4 Thomson ABR. Acute exposure of rabbit jejunum to ethanol. In vitro uptake of hexoses. Dig Dis Sci 1984: 29: 267-74.

5 Al-Balool FY. Effect of acute ethanol on the kinetics of electrogenic transfer of some amino acids and hexose sugars. Med Sci Re's 1987: 15: 395-6.

6 Mazzanti R. Debnam ES. Jenkins WJ. Effect of chronic ethanol intake on lactase activity and active galactose absorption in rat small intestine. (jut 1987: 28: 56-60). 
7 Mazzanti R. Jenkins WJ. Effect of chronic ethanol ingestion on enterocyte turnover in rat small intestine. Gut 1987; 28: 52-5.

8 Schultz SG. Sodium-coupled solute transport by small intestine: a status report. Am J Physiol 1977; 233: E249-54.

9 Ryle PR. Broillet A. Perrisoud D. et al. A comparative study of the effects of $(+)$ - catechin and 3-Palmitoyl$(+)$-catechin on alcoholic fatty liver in the rat. Alcohol Alcohol 1983; 18: 239-48.

10 Ananda Rao G. Larkin EC. Inadequate intake by growing rats of essential nutrients from liquid dicts used for chronic alcohol consumption. Nutr Res 1985: 5: 78996.

11 Debnam ES. Thompson CS. The effects of fasting on the potential difference across the brush border membrane of enterocytes in rat small intestine. $J$ Physiol $1984 ;$ 355: 449-56.

12 Debnam ES. Levin RJ. An experimental method of identifying and quantifying the active transfer electrogenic component from the diffusive component during sugar absorption measured in vivo. $J$ Physiol 1975; 246: 181-96.

13 Somogyi M. A new reagent for the determination of sugars. J Biol Chem 1945; 160: 61-8.

14 Pocock SJ. Clinical trials: a practical approach. Chichester: John Wiley, 1983: 213-6.

15 Okada Y, Sato T, Inouye A. Effects of potassium ions and sodium ions on membrane potential of epithelial cells in rat duodenum. Biochim Biophys Acta 1975; 413: 104-15.

16 Stewart CP, Turnberg LA. Glucose depolarises villus but not crypt cell apical membrane potential difference: a micropuncture study of crypt-villus heterogeneity in the rat. Biochim Biophys Acta 1987; 902: 293-300.

17 Wilson FA. Hoyumpa AM. Ethanol and small intestinal transport. Gastroenterology 1979; 76: 388-403.

18 Diamond JM, Karasov WH. Effect of dictary carbo- hydrate on monosaccharide uptake by mouse small intestine in vitro. J Physiol 1984; 349: 419-40.

19 Debnam ES. The kinetics of active, electrogenic hexose transport in the proximal and distal rat small intestine following overnight fasting. J Physiol 1978; 281: 38-39P.

20 Halsted C. Robles E. Mezcy E. Distribution of ethanol in the human gastrointestinal tract. Am J Clin Nutr 1973; 26: $831-4$.

21 Debnam ES. Effect of sodium concentration and plasma sugar concentration on hexose absorption by the rat jejunum in vivo. Pflugers Archiv 1982; 393: 104-8.

22 Debnam ES, Levin RJ. Influence of specific dictary sugars on the jejunal mechanisms for glucose, galactose and $\alpha$-methyl glucoside absorption: evidence for multiple sugar carriers. Gut 1976; 17: 92-9.

23 Barry RJC, Dikstein S, Matthews J, et al. Electrical potentials associated with intestinal sugar transfer. J Physiol 1964; 171: 316-38.

24 Cremaschi D, James PS, Meyer G, et al. Membrane potentials of differentiating enterocytes. Biochem Biophys Acta 1982; 688: 271-4.

25 Mazzanti R. Srai SKS, Debnam ES, et al. The effect of chronic ethanol consumption on iron absorption in rats. Alcohol Alcohol 1987; 22: 47-52.

26 Thompson CS, Debnam ES. Starvation-induced changes in the autoradiographic localisation of valine uptake by rat small intestinc. Experientia 1986; 42: 945-8.

27 Thompson CS, Debnam ES. Hyperglucagonacmia: Effects on active nutrient uptake by the rat jejunum. $J$ Endocrinol 1986; 111: 37-42.

28 Ballard HJ. Wilkes JM. Hirst BH. Effects of alcohols on gastric and small intestinal apical membranc integrity and fluidity. Gut 1988; 29: 1648-55.

29 Lindenbaum J, Lieber CS. Effects of chronic ethanol administration on intestinal absorption in man in the absence of nutritional deficiency. Ann NY Acad Sci 1975; 252: 228-34. 